\title{
Immigration and the City
}

\author{
by Eric Fong and Brent Berry \\ Cambridge (UK): Polity Press, 2017 \\ Immigration \& Society Series \\ ISBN 978-0-7456-9002-5 \\ Softcover $\$ 27.95,176 \mathrm{pp}$.
}

\section{Reviewed by Nikki Eller \\ University of Washington}

Immigration and the City by Eric Fong and Brent Berry provides a good overview of what has been studied and where current research is heading with respect to the ways immigrants are shaped by, and help to shape, their host country. Since so many immigrants to the US and Canada arrive and settle in large "gateway" cities, this book covers what is known about how immigrants interact with urban and suburban environments.

The book begins with an orientation to the theories of residential segregation, including the original Concentric Zone Model by Burgess, but also newer models of Spatial Assimilation, Place Stratification, and Individual Preference. The theories are grounded in empirical evidence from different types of neighbourhoods (mixed, gentrified, immigrant suburban, etc.), which suggests that the diversity of residential patterns may require diverse explanatory models. Another layer of complexity is added as social scientists begin to tackle the interactions of more than two groups in a neighborhood. Theories and terms discussed later in the book include classical, neoclassical, and segmented assimilation models, ethnic enclave, ethnic economy, and ethnoburb.

This finding of diverse and complex theories and evidence needed to explain the immigrant experience is repeated throughout Immigration and the City, reflecting the diversity of the population and places studied. While sweeping generalizations may not be possible, some patterns do emerge.

In the contexts of housing attainment and economic opportunity, Fong and Berry discuss ethnic communities as beneficial to recent immigrants for easing their transition, but possibly detrimental to long-term housing and employment, as isolation from the broader society may limit opportunities. For example, a recently arrived immigrant may be able to quickly obtain housing within an ethnic enclave, but have difficulty moving outside of the enclave later. Likewise, community pressure to hire co-ethnic members may make it easier for a recently arrived immigrant to find a job, but harder for an immigrant business owner to hire from outside the group.

Furthermore, though immigrant groups may have internal preferences when it comes to the types of housing and work they prefer, discrimination is clearly a factor that works against obtaining either outside an ethnic enclave. The empirical evidence shows that city context has a major influence on the economic achievement and locational distribution of immigrants, and discrimination may play a part in that. High rates of entrepreneurship found among immigrants are likely a consequence of both discrimination and selection bias from the immigration process. 
Although much of the research on immigrants has focused on their assimilation into the host society, Fong and Berry also cover how immigrants in turn shape their environment—specifically, the food, play, and landscapes. Although ethnic cuisine has been and continues to be integrated into mainstream culture, ethnic communities often maintain separate leisure activities. This can vary depending on a variety of factors, including time in country, SES, size of ethnic community, and affinity for particular activities; soccer is only one example of an activity that has increased in North American popular culture due to immigrant influence. Landscape changes can be observed in the increased demand for housing built to accommodate larger households, shopping centers built in accordance with immigrant tastes, and differential uses of public space.

Data from time-use surveys offer a window into the immigrant experience that may not be easily visible. Research shows that adults are likely to have non-standard work hours and long commutes, impeding their ability to engage in other activities, and that immigrant children are likely to spend more time studying or caring for other siblings than non-immigrant children. Gender differences in time use may also be more marked in immigrant households.

Overall, Immigration and the City is a good introduction to both the theory and empirical evidence that surrounds the urban immigrant experience, and describes how immigrants and cities help shape each other. The book's clear organization and concise style make it ideal for busy researchers and students alike. 